\title{
Direct feeding of the black hole at the Galactic Centre with radial gas streams from close-in stellar winds
}

\section{Citation}

Loeb, Abraham. 2004. "Direct Feeding of the Black Hole at the Galactic Centre with Radial Gas Streams from Close-in Stellar Winds." Monthly Notices of the Royal Astronomical Society 350 (2): 725-28. https://doi.org/10.1111/j.1365-2966.2004.07684.x.

\section{Permanent link}

http://nrs.harvard.edu/urn-3:HUL.InstRepos:41417379

\section{Terms of Use}

This article was downloaded from Harvard University's DASH repository, and is made available under the terms and conditions applicable to Other Posted Material, as set forth at http:// nrs.harvard.edu/urn-3:HUL.InstRepos:dash.current.terms-of-use\#LAA

\section{Share Your Story}

The Harvard community has made this article openly available. Please share how this access benefits you. Submit a story. 


\title{
Direct feeding of the black hole at the Galactic Centre with radial gas streams from close-in stellar winds
}

\author{
Abraham Loeb ${ }^{\star}$ \\ Astronomy Department, Harvard University, 60 Garden Street, Cambridge, MA 02138, USA
}

Accepted 2004 January 28. Received 2003 December 24

\begin{abstract}
We show that the recently discovered orbits of massive stars with closest approach of $\sim 10^{3}$ Schwarzschild radii from Sgr $\mathrm{A}^{*}$ allow winds from these stars to provide the required mass deposition rate near the black hole horizon. The observed luminosity of $\operatorname{Sgr~}^{*}$ does not require viscous transport of angular momentum, as long as the total wind mass loss rate reaches $\sim 10^{-6} \mathrm{M}_{\odot} \mathrm{yr}^{-1}$ from the close-in stars. The specific orbits of the nearest stars should cause modulation of the radio and infrared flux from Sgr $\mathrm{A}^{*}$ on a time-scale of years in a predictable fashion.
\end{abstract}

Key words: stars: winds, outflows - Galaxy: centre.

\section{INTRODUCTION}

Early in the development of accretion flow theory, it was realized that angular momentum can severely limit the accretion rate of gas on to a black hole relative to the spherical infall value (the so-called Bondi rate; Bondi 1952). Indeed, most of the hot gas revealed by recent X-ray observations within $\sim 0.1 \mathrm{pc}$ of the Galactic Centre (Baganoff et al. 2003) is apparently not flowing into Sgr A*, the massive black hole there (Quataert 2003). The latest models of the radiatively inefficient accretion flow around $\mathrm{Sgr} \mathrm{A}^{*}$ estimate the accretion rate of gas into the black hole horizon at $\sim 10^{-8} \mathrm{M}_{\odot} \mathrm{yr}^{-1}$, several orders of magnitude below the Bondi rate (Yuan, Quataert \& Narayan 2003). This low accretion rate is required by the frequency dependence of the linear polarization data (Bower et al. 2003), in combination with the synchrotron radio flux and the recently detected infrared emission from Sgr A* (Genzel et al. 2003b; Ghez et al. 2004). The absolute minimum accretion rate required by the bolometric luminosity of $\operatorname{Sgr}^{*}\left(\sim 10^{36} \mathrm{erg} \mathrm{s}^{-1}\right)$ is $\sim 2 \times 10^{-10} \mathrm{M}_{\odot} \mathrm{yr}^{-1}$ $(\epsilon / 0.1)^{-1}$, where $\epsilon$ is the radiative efficiency of the accreting mass.

The disparity between the large gas reservoir available at the outer radius of influence of $\mathrm{Sgr} \mathrm{A}^{*}$ and the small mass that actually flows into its horizon has inspired a variety of accretion models in which the mass inflow rate diminishes with decreasing radius, as a result of convection (Quataert \& Gruzinov 2000a,b; Narayan, Igumenshchev \& Abramowicz 2000), turbulence (Coker \& Melia 1997; Pen, Matzner \& Wong 2003), or outflows (Blandford \& Begelman 1999; Yuan, Markoff \& Falcke 2002). Here we highlight the significance of an important ingredient that was omitted by these models, namely the powerful winds from known stars that are embedded within the dilute accretion flow towards Sgr A*.

Sgr A* was recently found to be surrounded by a cluster of young, massive stars. Over the past year, three of these stars, named SO-2,

^E-mail: aloeb@cfa.harvard.edu
SO-16 and SO-19 by Ghez et al. (2004, see parallel work by Schödel et al. 2003), were inferred to have orbits with pericentric distances of $\sim 100$ au or $\sim 10^{3}$ Schwarzschild radii from the central black hole. The detection of a $\mathrm{Br} \gamma$ and $\mathrm{He}$ I features in the spectrum of SO-2 indicates that it is probably an $\mathrm{O} 8-\mathrm{B} 0$ star, while the other bright stars are also likely to be OB stars, consistent with the lack of a CO absorption feature in their spectra (Ghez et al. 2003a; Eisenhauer et al. 2003).

The winds of $\mathrm{O}$ stars on the main sequence have typical mass loss rates of $\dot{M}_{\mathrm{w}}=10^{-7}-10^{-6} \mathrm{M}_{\odot} \mathrm{yr}^{-1}$ and speeds in the range $v_{\mathrm{w}}=$ $1-3 \times 10^{3} \mathrm{~km} \mathrm{~s}^{-1}$ (Puls et al. 1996; Repolust, Puls \& Herrero 2004). In Section 2.1, we show that SO-2, SO-16 and SO-19 can supply the required low angular momentum gas that fuels the emission from Sgr $\mathrm{A}^{*}$ without requiring viscous transport of angular momentum. To demonstrate this point, we adopt the simplest model in which the radial gas streams move on ballistic Keplerian orbits. Gas with higher angular momentum is assumed, for simplicity, to be driven out of the system, as inferred to be case at the Bondi radius (Quataert 2003). In Section 2.3 we show that the characteristic ram pressure of the radial streams is well above the thermal pressure of the diffuse hot gas detected by the Chandra X-ray Observatory (Baganoff et al. 2003), making the ballistic approach viable. In Section 2.2 we illustrate how the specific orbital parameters of the close-in stars can be used to predict the long-term modulation of the radio and infrared flux of Sgr A*. Finally, we discuss the implications of these results in Section 3.

\section{DIRECT FLOW OF GAS INTO THE BLACK HOLE HORIZON}

\subsection{Method of calculation}

We consider the simplest model in which the gas elements move on ballistic Keplerian orbits. This assumption is justified if: 


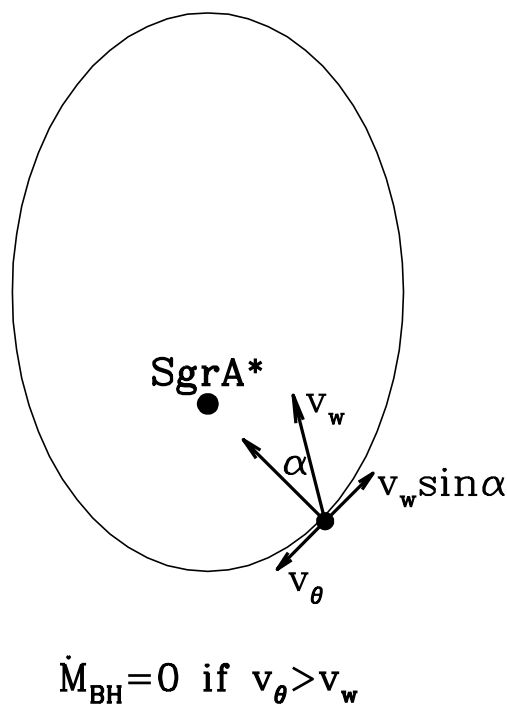

Figure 1. Schematic geometry of a radial stream. The stream is confined to a narrow cone centred on the direction where the tangential component of the wind velocity, $v_{\mathrm{w}} \sin \alpha$, cancels the tangential velocity of the star in its orbit around Sgr A*, $v_{\theta}$ (see equation 2). The small opening angle of the radial stream, $\delta$, is dictated by the maximum angular momentum, $J_{\max }$, that allows the gas to join the compact emission region around $\mathrm{Sgr} \mathrm{A}^{*}$ (equation 3).

(i) viscosity is sufficiently weak that mainly gas on nearly radial orbits approaches the horizon of $\mathrm{Sgr} \mathrm{A}^{*}$; (ii) the inflow of low angular momentum gas is dominated by one stellar wind at a time so that multiple gas streams do not collide; and (iii) the wind ram pressure is much higher than the gas into which it propagates, so that the low angular momentum gas is not deflected by hydrodynamic forces on its radial trajectory towards the black hole horizon. We justify the latter two conditions in Section 2.3.

The mass of gas directed into the compact emission region around the black hole horizon is the sum of all gas elements sent out from a stellar wind with angular momentum per unit mass smaller than some critical value, $J_{\max }$ (see Fig. 1). We write

$J_{\max }=\eta\left(\frac{4 G M}{c}\right)$

where $M=4 \times 10^{6} \mathrm{M}_{\odot}$ is the black hole mass (Ghez et al. 2003b; Schödel et al. 2003), and $4 G M / c$ is the maximum angular momentum with which the gas would enter directly into the horizon of a Schwarzschild black hole (see equation 14.2.12 in Shapiro \& Teukolsky 1983). For a nearly radial trajectory starting at the star, we find that

$\eta=0.5 x /(x-1)^{1 / 2}$,

where $x=r_{\min } /\left(2 G M / c^{2}\right)$ is the distance of closest approach to Sgr A* (in Schwarzschild radius units) of the ballistic gas orbit. ${ }^{1}$ In popular emission models (Melia, Liu \& Coker 2001a; Melia et al. 2001b; Liu \& Melia 2002a; Yuan et al. 2002, 2003), most of the bolometric luminosity of Sgr $\mathrm{A}^{*}$ is produced within $\sim 30$ Schwarzschild radii, implying $\eta \lesssim 2.8$ for the wind gas to join the emission region directly from its orbit. Next, let us calculate the fraction of the wind material that has an orbital angular momentum $|J|<J_{\max }$ for a star at radius $r$ in an orbit with eccentricity $e$ and semimajor axis $a$.

${ }^{1}$ This result is obtained by setting $\tilde{E} \approx 1$ and solving $V(r)=\tilde{E}$ in equation (12.4.25) of Shapiro \& Teukolsky (1983).

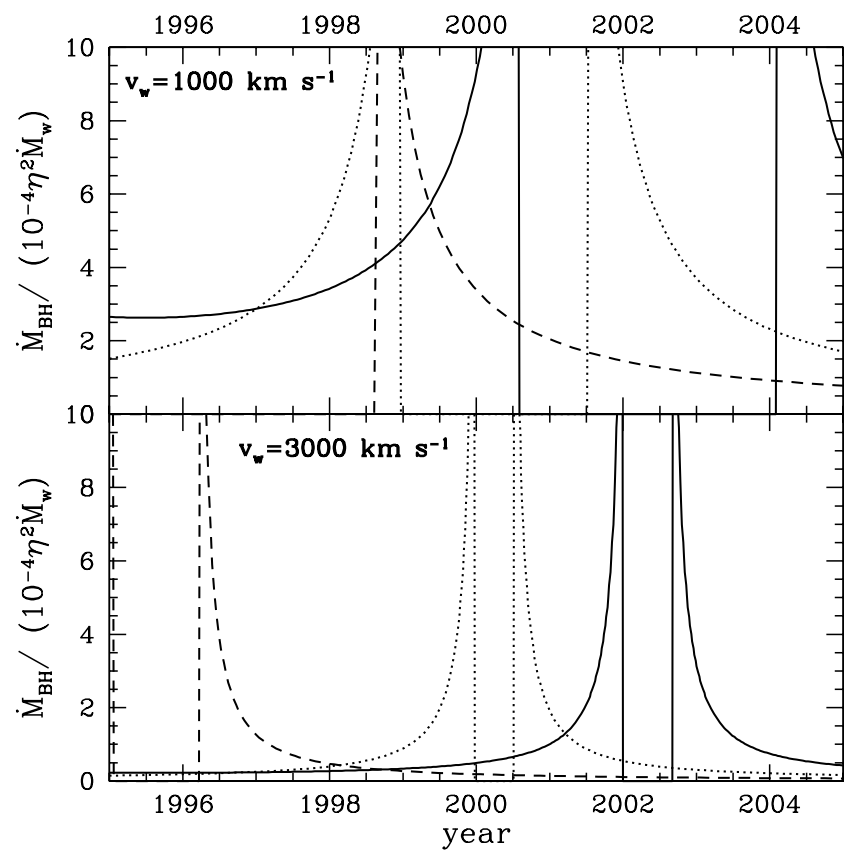

Figure 2. Mass deposition rate into the emission region surrounding the horizon of the black hole at the Galactic Centre, $\dot{M}_{\mathrm{BH}}$, as a function of calendar year. The various line types correspond to the individual contributions of the three bright stars nearest to Sgr A*, namely SO-2 (solid), SO-16 (dashed) and SO-19 (dotted). The orbital parameters of these stars were adopted from table 3 of Ghez et al. (2003b). The sensitivity of the results to the assumed wind velocity, $v_{\mathrm{w}}$, is illustrated by the difference between the top $\left(v_{\mathrm{w}}=\right.$ $\left.1000 \mathrm{~km} \mathrm{~s}^{-1}\right)$ and bottom $\left(v_{\mathrm{w}}=3000 \mathrm{~km} \mathrm{~s}^{-1}\right)$ panels. The gaps in $\dot{M}_{\mathrm{BH}}$ occur during phases with $v_{\theta}>v_{\mathrm{w}}$ along the stellar orbit, and are centred on pericentre passages.

The low angular momentum gas is confined to a narrow cone centred on an angle $\alpha$ relative to the direction of Sgr A* (see Fig. 1), such that

$v_{\theta}+v_{\mathrm{w}} \sin \alpha=0$,

where $v_{\mathrm{w}}$ is the wind speed, $v_{\theta}=\ell / r$ is the tangential component of the orbital velocity of the star [using the polar coordinates $(r, \theta)$ in the orbital plane], and

$\ell=\left[G M a\left(1-e^{2}\right)\right]^{1 / 2}$

is the orbital angular momentum of the star. Note that condition (2) may be satisfied only through a portion of the stellar orbit that is sufficiently far from $\mathrm{Sgr} \mathrm{A}^{*}$ that $v_{\mathrm{w}}$ is larger than $v_{\theta} \propto r^{-1}$; elsewhere, we set the mass deposition rate into Sgr $\mathrm{A}^{*}$ to zero (see the gaps of vanishing $\dot{M}_{\mathrm{BH}}$ in Fig. 2). The small opening angle of the Sgr $\mathrm{A}^{*}$ acceptance cone, $\delta \ll \alpha$, is defined by the condition

$v_{\theta}+v_{\mathrm{w}} \sin (\alpha+\delta)=\frac{J_{\max }}{r}$.

Using the small-angle approximation, that is $\sin (\alpha+\delta) \approx \sin \alpha+$ $\delta \cos \alpha$, we find

$\delta=\frac{J_{\max }}{v_{\mathrm{w}} r \cos \alpha}$,

where $\cos \alpha=\left[1-\left(\ell / v_{\mathrm{w}} r\right)^{2}\right]^{1 / 2}$.

We can now evaluate the fraction of the wind mass that is channelled through the acceptance cone of the emission region around the black hole horizon. Assuming that the wind outflow is 
spherically symmetric in the star rest frame, this fraction is

$\frac{\dot{M}_{\mathrm{BH}}}{\dot{M}_{\mathrm{w}}}=\frac{\pi \delta^{2}}{4 \pi}=\left(\frac{J_{\mathrm{max}}}{2 v_{\mathrm{w}} r}\right)^{2}\left(1-\frac{\ell^{2}}{v_{\mathrm{w}}^{2} r^{2}}\right)^{-1}$.

For the characteristic values of $v_{\mathrm{w}} \sim 1000 \mathrm{~km} \mathrm{~s}^{-1}$ and $r \sim 10^{3} \mathrm{au}$, one gets $\dot{M}_{\mathrm{BH}} \sim 5 \times 10^{-3}(\eta / 3)^{2} \dot{M}_{\mathrm{w}}$.

If the gas element leaves the star at a time $t$, then it will reach the black hole on a radial orbit at a time $t+\Delta t$. The time delay $\Delta t$ can be easily calculated from the initial radial distance of the star $r$, and the initial radial velocity of the gas,

$u_{r}=\frac{\mathrm{d} r}{\mathrm{~d} t} \pm v_{\mathrm{w}}\left[1-\left(\frac{\ell}{v_{\mathrm{w}} r}\right)^{2}\right]^{1 / 2}$,

where there are either zero, one or two allowed cones under the restriction that $u_{r}<0$. The time-scale for the gas to be drained into the black hole horizon from the compact emission region around it (Hawley, Balbus \& Stone 2001) is expected to be short compared to the time-scales of interest here and so we neglect it.

\subsection{Numerical results}

Fig. 2 shows the numerical solution of equations (5) and (6) for $\dot{M}_{\mathrm{BH}}$ as a function of $t+\Delta t$, given the orbital parameters of SO2 (solid), SO-16 (dashed) and SO-19 (dotted), as summarized in table 3 of Ghez et al. (2003b). The evolution of the mass deposition rate into the black hole horizon is presented for the values $v_{\mathrm{w}}=1 \times$ $10^{3} \mathrm{~km} \mathrm{~s}^{-1}$ (upper panel) and $3 \times 10^{3} \mathrm{~km} \mathrm{~s}^{-1}$ (lower panel), which bracket the range of wind speeds deduced from observed spectra of O stars (Puls et al. 1996; Repolust et al. 2004).

For the value of $\dot{M}_{\mathrm{w}} \sim 10^{-6} \mathrm{M}_{\odot} \mathrm{yr}^{-1}$ found in bright Galactic O stars on the main sequence (Puls et al. 1996; Repolust et al. 2004), we get a total feeding rate of $\sim 10^{-8}(\eta / 3)^{2} \mathrm{M}_{\odot} \mathrm{yr}^{-1}$, comparable to the value required by the polarization data in accretion models of Sgr A* (Melia, Liu \& Coker 2000; Bromley, Melia \& Liu 2001; Yuan et al. 2003). Most of the wind material spreads over the highly eccentric orbit of its parent star, and so we find that $\dot{M}_{\mathrm{w}} \lesssim 10^{-6} \mathrm{M}_{\odot} \mathrm{yr}^{-1}$ for SO-2, SO-16 or SO-19 yields electron densities below the canonical inflow model of Yuan et al. (2003) with the resultant Faraday rotation measure satisfying current polarization constraints.

It is natural to expect the luminosity of Sgr A* to scale with $\dot{M}_{\mathrm{BH}}^{\beta}$, where $\beta$ ranges between 1 (for disc or jet emission with a constant radiative efficiency) and 2 (for radiatively inefficient flows). The emission spectrum can be generically explained by synchrotron and inverse Compton processes (Melia \& Falcke 2001; Yuan et al. 2002, 2003) in a hot, magnetized gas within tens of Schwarzschild radii from Sgr A*, independent of the exact accretion geometry. The radial streams that reach this region would likely dissipate a fraction of their kinetic energy through shocks, and settle into a rotationally supported configuration around Sgr A* [see Hawley et al. (2001), for the subsequent evolution of such as torus]. The interaction of the freely falling material with the rotating torus may resemble the situation in wind-fed X-ray binaries (Beloborodov \& Illarionov 2001). The magnetic fields carried by the infalling streams could be amplified by plasma instabilities and produce the synchrotron luminosity of Sgr A*.

\subsection{Are ballistic orbits plausible?}

The properties of the accretion flow under consideration here resemble the conditions in wind-fed X-ray binaries (Illarionov \& Beloborodov 2001, and references therein). The accreting matter in such binaries originates in a wind from an OB star with similar mass loss rates to those assumed here, and is thought to fall freely towards the boundary of the accretor (be it the surface of a neutron star or the horizon of a black hole) where the X-rays are produced. The ballistic approach adopted here has been used successfully to derive the accretion rate and the corresponding X-ray luminosity of accreting neutron stars (Illarionov \& Beloborodov 2001).

Fig. 2 illustrates that at most times there is only one gas stream dominating the mass deposition rate into $\mathrm{Sgr} \mathrm{A}^{*}$. Collisions of multiple streams with comparable momentum flux are rare. The dominant gas streams follow ballistic orbits as long as their ram pressure, $p_{\mathrm{w}}=\rho_{\mathrm{w}} v_{\mathrm{w}}^{2}$, exceeds the thermal pressure of the dilute gas through which they propagate, $p_{\text {th }}$. We find

$p_{\mathrm{w}}=\frac{\dot{M}_{\mathrm{w}} v_{\mathrm{w}}}{4 \pi d^{2}}=3 \times 10^{3} \dot{M}_{\mathrm{w},-6} v_{\mathrm{w}, 2}\left(\frac{10^{3} \mathrm{au}}{d}\right)^{2} \frac{\mathrm{keV}}{\mathrm{cm}^{3}}$,

where we write $\dot{M}_{\mathrm{w},-6} \equiv\left(\dot{M}_{\mathrm{w}} / 10^{-6} \mathrm{M}_{\odot} \mathrm{yr}^{-1}\right), v_{\mathrm{w}, 2} \equiv\left(v_{\mathrm{w}} /\right.$ $2000 \mathrm{~km} \mathrm{~s}^{-1}$ ) and $d$ is the distance from the star (ignoring gravitational focusing by Sgr $\mathrm{A}^{*}$ ). The diffuse X-ray-emitting gas, spread over a scale of $\gtrsim 10^{4}$ au from Sgr A*, has a characteristic temperature of $\sim 1.3 \mathrm{keV}$ and a density $\sim 30 \mathrm{~cm}^{-3}$ (Baganoff et al. 2003), implying a background thermal pressure, $p_{\text {th }} \sim 40 \mathrm{keV} \mathrm{cm}^{-3}$, which is below the ram pressure of the radially infalling streams at $r \ll$ $10^{4}$ au. As each stream falls towards the black hole, it gets accelerated and compressed and so its ram pressure increases. Since its infall speed scales as $\propto r^{-1 / 2}$ and its surface area must decrease as $r^{-2}$, we expect that the ram pressure would scale as $p_{\mathrm{w}} \propto r^{-3}$ and that the condition $p_{\mathrm{w}}>p_{\text {th }}$ will be maintained throughout the trajectory of the stream. Our simplifying assumption (which we intend to examine critically with a direct numerical simulation in the future) is that most of the outer gas detected by Chandra or any other gas with significant angular momentum is not able to flow inwards similarly to the radial streams in the absence of efficient angular momentum transport. Note that a clumpy wind would enhance the survivability of the radial streams and potentially lead to short-term variability of Sgr A*.

The fate of wind gas with angular momentum above $J_{\max }$ remains an interesting open question. The linear polarization data (Bower et al. 2003) imply that most of this gas does not reach the vicinity of the black hole. Since most of the energy dissipation occurs at $\sim 10$ Schwarzschild radii and the gravitational binding energy per particle decreases considerably at larger radii, we conjecture that heat flux from the inner regions unbinds the outer gas and expels it in an outflow [similarly to the conditions at the Bondi radius (see Quataert 2003)].

\section{DISCUSSION}

We have found that radial gas streams from the winds of massive stars with recently discovered orbits that approach within $\sim 10^{3}$ Schwarzschild radii of the black hole at the Galactic Centre can supply the required mass flow near the central black hole horizon and power the radio and infrared emission of Sgr $\mathrm{A}^{*}$. For O star winds with a total $\dot{M}_{\mathrm{w}} \sim 10^{-6} \mathrm{M}_{\odot} \mathrm{yr}^{-1}$, we get that SO-2, SO-16 and SO-19 can supply as much as $\sim 10^{-8}(\eta / 3)^{2} \mathrm{M}_{\odot} \mathrm{yr}^{-1}$ of gas in orbits directed straight into the emission region around the black hole horizon. The total mass inflow rate would increase if angular momentum of other gas elements is transported away by magnetic or turbulent stresses. The surprising existence of massive stars in orbits with closest approach distances of $\sim 100$ au poses a major puzzle to theories of star formation (Genzel et al. 2003a), and was 
not incorporated in previous modelling of the gas flow around Sgr A* (e.g. Coker \& Melia 1997; Coker, Melia \& Falcke 1999; Yuan et al. 2003).

The parameters of the winds could in principle be determined through detailed spectroscopic observations of the stars in the Sgr A* cluster. Once the wind properties of the individual stars are constrained, our accretion model will be easily testable since it correlates the long-term ( $>$ month) variability of the radio or infrared emission from $\mathrm{Sgr} \mathrm{A}^{*}$ with the orbital phases of the stars around it (see Fig. 1). The simplified calculation presented here can be improved using a full hydrodynamic simulation and compared against observational data on the long-term variability of Sgr A* (Zhao et al. 2003) for different choices of $v_{\text {w }}$ and $\dot{M}_{\text {w }}$ per individual star [for an alternative source of this variability, see Liu \& Melia (2002b)]. A proper numerical treatment is challenging since only a small fraction of the gas mass follows the radial orbits of interest here, and since the inflow spans several orders of magnitude in radius - requiring a large dynamic range in spatial and temporal resolution. Previous accretion simulations, such as those by Coker \& Melia (1997), Proga \& Begelman (2003) or Rockefeller et al. (2004), did not meet the demanding requirements of this accretion problem.

Sgr A* may be surrounded by fainter stars that have not been detected so far, even though they dominate the steady mass flow into the black hole. However, winds from massive stars are driven by radiation pressure, and so the stars dominating the fluctuations in the mass flow into the black hole horizon should have the highest luminosities and be included in the current census of bright infrared sources near Sgr A*. Massive stars that are much further away also possess powerful winds (Krabbe et al. 1991; Najarro et al. 1997; Coker \& Melia 1997; Quataert 2003), but their individual contributions to the radial mass flux near the horizon of Sgr A* is reduced compared to the close-in stars, ${ }^{2}$ since $\dot{M}_{\mathrm{BH}} \propto r^{-2}$ in equation (5). For example, IRS $16 C$ with its $\dot{M}_{\mathrm{w}} \approx 8 \times 10^{-5} \mathrm{M}_{\odot} \mathrm{yr}^{-1}$ and $v_{\mathrm{w}} \sim 650 \mathrm{~km} \mathrm{~s}^{-1}$ at $r \gtrsim 2 \times 10^{4} \mathrm{au}$, and IRS $13 \mathrm{E} 1$ with its $\dot{M}_{\mathrm{w}} \approx 8 \times 10^{-4} \mathrm{M}_{\odot} \mathrm{yr}^{-1}$ and $v_{\mathrm{w}} \sim 1000 \mathrm{~km} \mathrm{~s}^{-1}$ at $r \gtrsim 4 \times 10^{4}$ au (Quataert, Narayan \& Reid 1999), produce at most a radial mass flux comparable to that of SO-2 if the latter has $\dot{M}_{\mathrm{w}} \sim 10^{-6} \mathrm{M}_{\odot} \mathrm{yr}^{-1}$. Nevertheless, the steady mass flux into $\mathrm{Sgr} \mathrm{A}^{*}$ may still be dominated by undetected stars or by gas that originates at large distances and loses its angular momentum through viscous transport (Yuan et al. 2003). In this case, the winds from the close-in stars would only modulate a fraction of $\dot{M}_{\mathrm{BH}}$, but their fractional contribution could still be identified through the predictable short-term variability that their orbits introduce to the luminosity of Sgr A*.

\section{ACKNOWLEDGMENTS}

I thank Jean-Pierre Lasota, Eric Pfahl, Eliot Quataert, Mark Reid, George Rybicki and Eli Waxman for comments on the manuscript.

\footnotetext{
${ }^{2}$ Statistically, the net mass flux is dominated by the close-in stars as long as the number density run of wind sources is steeper than $\propto r^{-1}$, as is the case around Sgr A* (Genzel et al. 2003a).
}

This work was supported in part by NASA grant NAG 5-13292, and by NSF grants AST-0071019 and AST-0204514.

\section{REFERENCES}

Baganoff F. K. et al., 2003, ApJ, 591, 891

Beloborodov A. M., Illarionov A. F., 2001, MNRAS, 323, 167

Blandford R. D., Begelman M. C., 1999, MNRAS, 303, L1

Bondi H., 1952, MNRAS, 112, 195

Bower G. C., Wright M. C. H., Falcke H., Backer D. C., 2003, ApJ, 588, 331

Bromley B. C., Melia F., Liu S., 2001, ApJ, 555, L83

Coker R., Melia F., 1997, ApJ, 488, L149

Coker R., Melia F., Falcke H., 1999, ApJ, 523, 642

Eisenhauer F., Schödel R., Genzel R., Ott T., Tecza M., Abuter R., Eckart A., Alexander T., 2003, ApJ, 597, L121

Genzel R. et al., 2003a, ApJ, 594, 812

Genzel R., Schödel R., Ott T., Eckart A., Alexander T., Lacombe F., Rouan D., Aschenbach B., 2003b, Nat, 425, 934

Ghez A. M. et al., 2003a, ApJ, 586, L127

Ghez A. M., Salim S., Hornstein S. D., Tanner A., Morris M., Becklin E. E., Duchene G., 2003b, ApJ, submitted (astro-ph/0306130)

Ghez A. M. et al., 2004, ApJ, 601, L159

Hawley J. F., Balbus S. A., Stone J. M., 2001, ApJ, 554, L49

Illarionov A. F., Beloborodov A. M., 2001, MNRAS, 323, 159

Krabbe A., Genzel R., Drapatz S., Rotaciuc V., 1991, ApJ, 382, L19

Liu S., Melia F., 2002a, ApJ, 566, L77

Liu S., Melia F., 2002b, ApJ, 573, L23

Melia F., Falcke H., 2001, ARA\&A, 39, 309

Melia F., Liu S., Coker R., 2000, ApJ, 545, L117

Melia F., Liu S., Coker R., 2001a, ApJ, 553, 146

Melia F., Bromley B. C., Liu S., Walker C. K., 2001b, ApJ, 554, L37

Najarro F., Krabbe A., Genzel R., Lutz D., Kudritzki R. P., Hillier D. J., 1997, A\&A, 325, 700

Narayan R., Igumenshchev I. V., Abramowicz M. A., 2000, ApJ, 539, 798

Pen U., Matzner C. D., Wong S., 2003, ApJ, 596, L207

Proga D., Begelman M. C., 2003, ApJ, 582, 69

Puls J. et al., 1996, A\&A, 305, 171

Quataert E., 2003, ApJL, submitted (astro-ph/0310446)

Quataert E., Gruzinov A., 2000a, ApJ, 539, 809

Quataert E., Gruzinov A., 2000b, ApJ, 545, 842

Quataert E., Narayan R., Reid M. J., 1999, ApJ, 517, L101

Repolust T., Puls J., Herrero A., 2004, A\&A, 415, 349

Rockefeller G., Fryer C. L., Melia F., Warren M. S., 2004, ApJ, in press (astro-ph/0309497)

Schödel R., Ott T., Genzel R., Eckart A., Mouawad N., Alexander T., 2003, ApJ, 596, 1015

Shapiro S. L., Teukolsky S. A., 1983, Black Holes, White Dwarfs, and Neutron Stars. Wiley, New York

Yuan F., Markoff S., Falcke H., 2002, A\&A, 383, 854

Yuan F., Quataert E., Narayan R., 2003, ApJ, 598, 301

Zhao J., Young K. H., Herrnstein R. M., Ho P. T. P., Tsutsumi T., Lo K. Y., Goss W. M., Bower G. C., 2003, ApJ, 586, L29

This paper has been typeset from a $\mathrm{T}_{\mathrm{E}} \mathrm{X} / \mathrm{L} \mathrm{T}_{\mathrm{E}} \mathrm{X}$ file prepared by the author. 